\title{
Western blot: proteins separating technique, protocol, theory and trouble shooting
}

\section{Abstract}

Western blotting is used to visualize proteins that have been separated by gel electrophoresis. It is an important technique used in cell and molecular biology. Specific proteins can be identified from a complex mixture of proteins extracted from cells. The western blot includes three major steps:
A. Separation by size
B. Transfer to a solid support and
C. Marking target protein using a proper primary and secondary antibody to visualize

This technical article will attempt to explain the technique in detail to help researcher in getting good results and theory behind western blot, and offer some ways to troubleshoot.

Keywords: molecular biology, proteins, antibody, gel electrophoresis, western blot, phosphate buffered saline, spectrophotometer, solidification, electro phorator, protein extraction, antibody, protocol, protease degradation, horse radish peroxidase, positive electrode, blotting membrane, concentration

\author{
Volume 4 Issue 2 - 2017 \\ Javed lqbal,' Riaz Ahmad² \\ 'School of Life Sciences, Shaanxi Normal University, China \\ ${ }^{2}$ Lab of biochemical technique and neurosciences, Shaanxi \\ Normal University, China \\ Correspondence: Javed lqbal, School of Life Sciences, Shaanxi
Normal University, Xian, P.R China, \\ Email imjavedkhan89@gmail.com
}

Received: November 03, 2016 | Published: March 08, 2017
Abbreviations: PBS, phosphate buffered saline; PVDF, poly vinylidene fluoride; BSA, bovine serum albumin; HRP, horse radish peroxidase

\section{Introduction}

Western blot is used in molecular and biochemical research to separate and identify the proteins of interest. This technique involve separation of mixture of proteins based on molecular weight, and thus by type, through gel electrophoresis. The results on gel are then transferred to a membrane producing a band for each protein. The membrane is then incubated with labels primary and secondary antibodies specific to the protein of interest. The unbound antibody from the membrane is washed off leaving only the bound antibody to the protein of interest. The bound antibodies are then detected by developing the film. As the antibodies specifically only bind to the target protein, only one band should be visible. The thickness of the band corresponds to the amount of protein present in the sample; thus doing a standard can indicate the amount of protein present. This paper will first describe the protocol in detail for western blot, accompanied by theory to rationalize the protocol and followed by the theoretical explanation of the procedure, and in the later section, troubleshooting tips for common problems.

\section{Technique}

\section{Protein extraction}

Protein can be extracted from different kind of samples, such as tissue or cells. Below is the protocol to extract proteins from adherent cells.

Adherent cells:

a. Washing cells in the tissue culture flask or dish by adding cold phosphate buffered saline (PBS) and shaking gently. Discard PBS. (Tip: Keep tissue culture dish on ice throughout).

b. Add PBS tissue culture flask or dish and use a cell scraper to dislodge the cells. Transfer the mixture into micro centrifuge tubes via Pipette.

c. Centrifuge at 1500 RPM for 5-10minutes and discard the supernatant.

d. Add $180 \mu \mathrm{L}$ of ice cold cell lysis buffer with $20 \mu \mathrm{L}$ fresh protease inhibitor cocktail. (Tip: If protein concentration is not high enough at the end, it is advised to repeat the procedure with a higher proportion of protease inhibitor cocktail).

e. Incubate for 30 minutes on ice, and then spinning for 10 minutes at $12,000 \mathrm{RPM}$, at $4^{\circ} \mathrm{C}$.

f. Transfer supernatant to a fresh tube and store on ice or frozen at $-20^{\circ} \mathrm{C}$ or $-80^{\circ} \mathrm{C}$.

g. Measure the concentration of protein using a spectrophotometer.

\section{Sample preparation}

i. Determine the volume of protein extract to ensure $50 \mu \mathrm{g}$ in each well (concentration=mass/volume).

ii. Add $5 \mu \mathrm{L}$ sample buffer to the sample, and make the volume in each lane equalized using dd H2O. Mix well. (Tip: Total volume of $15 \mu \mathrm{L}$ per lane is suggested).

iii. Heat the samples with dry plate for 5 minutes at $100^{\circ} \mathrm{C}$.

iv. After preparing in Table 1 the $10 \%$ stacking gel solution and separating gel solution, assemble the rack for gel solidification Figure 1. 
v. First add stacking gel solution carefully until the level is equal to the red bar holding the glass plates Figure 2. Add $\mathrm{H} 2 \mathrm{O}$ to the top. Wait for 20-30minutes until the gel turning solidified.

vi. Overlay the stacking gel with the separating gel, after removing the water. (Tip: It is better to tilt the apparatus and use a paper towel to remove the water).

vii. Insert the comb, ensuring that there are no air bubbles in stacking and separating gel Figure 3.

viii. Wait until the gel is solidified. Once the gel is solidified, it can be used for electrophoresis (Tip: Solidification can be easily checked by leaving some gel solution in a tube).

Table I Preparation of gel

\begin{tabular}{lll}
\hline Reagents & $\mathbf{1 0 \%}$ gel & $\mathbf{5 \%}$ gel \\
\hline $\mathrm{dH}_{2} \mathrm{O}$ & $2.925 \mathrm{ml}$ & $2.720 \mathrm{ml}$ \\
$30 \%$ Acr $/$ Bris & $2.550 \mathrm{ml}$ & 0.68 \\
Tris (PH8.8 I.5m) & $1.875 \mathrm{ml}$ & $0.520 \mathrm{ml}(\mathrm{PH} \mathrm{6.8)}$ \\
$10 \% \mathrm{SDS}$ & $75 \mathrm{ul}$ & $40 \mathrm{ul}$ \\
$10 \%$ AP & $75 \mathrm{ul}$ & $40 \mathrm{ul}$ \\
TEMED & $6 \mathrm{ul}$ & $4 \mathrm{ul}$
\end{tabular}

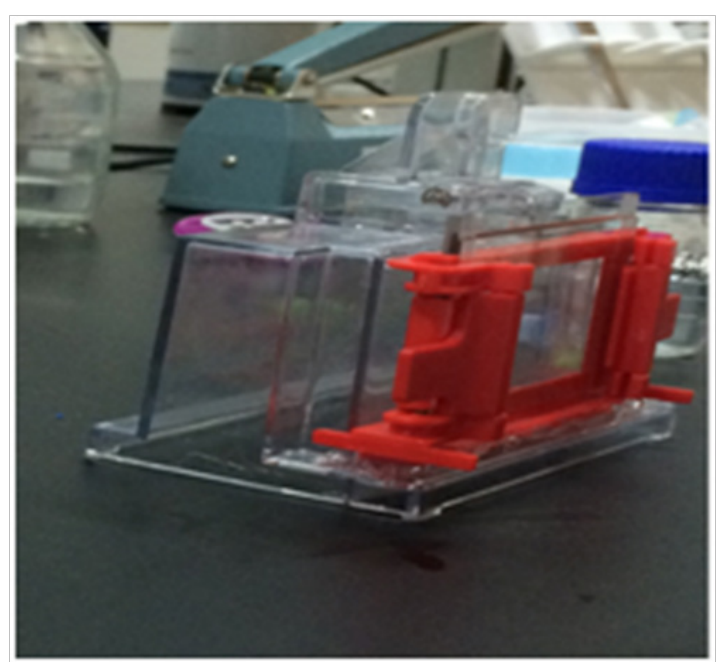

Figure I Assembled rack for gel solidification.

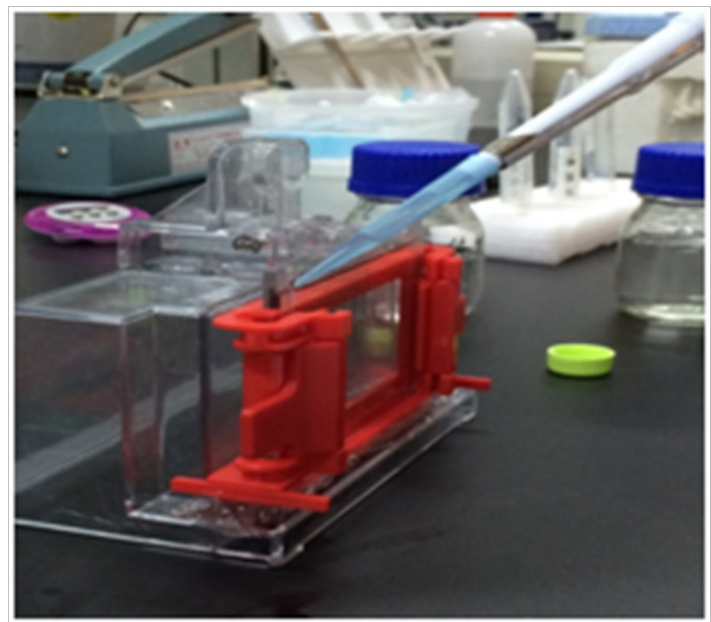

Figure 2 Add gel solution using a transfer pipette.

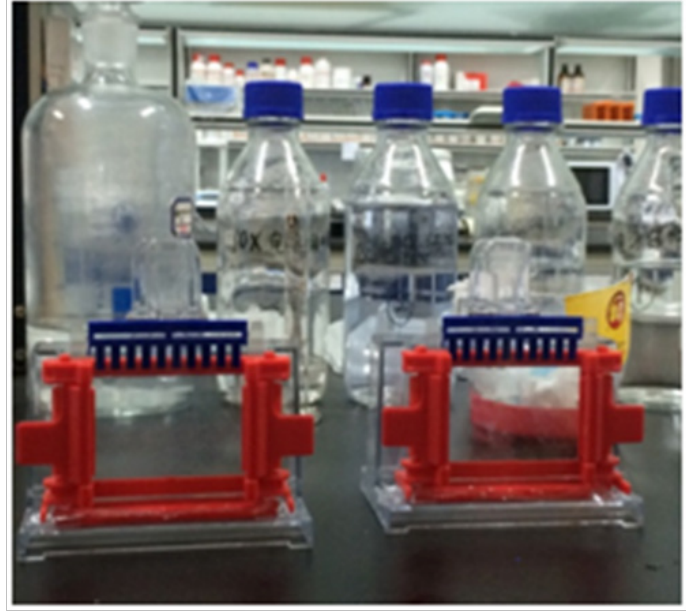

Figure 3 Placing comb after gel solution

\section{Gel electrophoresis}

a. For running the gel, pour the running buffer into the electro phorator.

b. Place gel inside the electro phorator and connect to a power supply. (Tip: When connecting to the power source always make sure to connect red to red, and black to black).

c. Make sure buffer covers the gel completely, and remove the comb carefully.

d. Load marker $(5 \mu \mathrm{L})$ followed by samples $(15 \mu \mathrm{L})$ into each well Figure 4.

e. Run the gel first with low voltage $(60 \mathrm{~V})$ for separating gel; then use higher voltage (140V) for stacking gel Figure 5a \& 5b.

f. Run the gel for approximately an hour, or until the dye front runs to the bottom of the gel Figure 6.

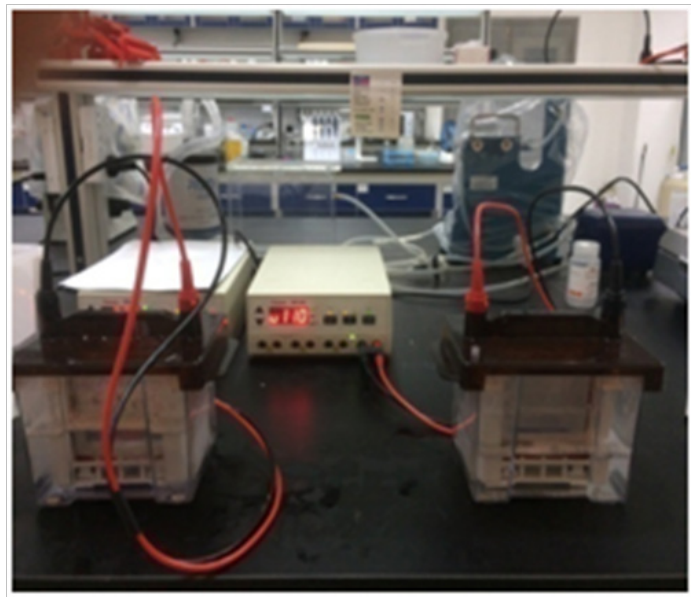

Figure 4 Add samples and molecular marker to the gel, after removing the combs and running on ice.

\section{Electro transfer from gel to PVDF membrane}

a. Cut 6 filter sheets and one poly vinylidene fluoride (PVDF) membrane to fit the measurement of the gel with the same dimensions.

b. Wet the sponge and filter paper in transfer buffer, and wet the PDVF membrane in methanol. 
c. Separate glass plates and retrieve the gel. After then, create a transfer sandwich as follows:
i. Sponge
ii. 3 Filter Papers
iii. Gel
iv. PVDF
v. 3 Filter Papers
vi. Sponge

(Tip: Ensure there are no air bubbles between the gel and PVDF membrane, and squeeze out extra liquid).

a. Relocate the transfer sandwich to the transfer apparatus placed on ice to maintain $4^{\circ} \mathrm{C}$. Add transfer buffer to the apparatus making sure the sandwich is covered with the buffer. Place electrodes on top of the sandwich, ensuring that the PVDF membrane is between the gel and a positive electrode Figure 7.

b. Transfer for 80-90minutes Figure 8. (Tip: The running time should be proportional to the thickness of the gel, so this may be reduced to 45 minutes for $0.75 \mathrm{~mm}$ gels).

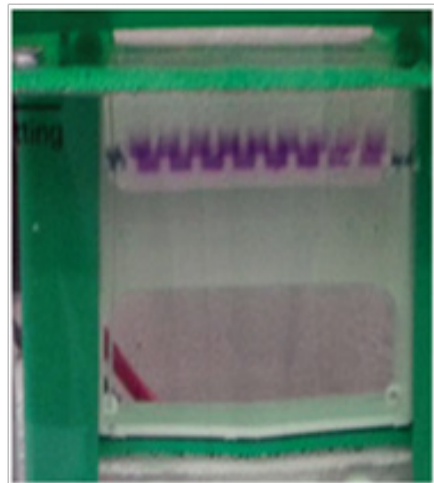

(a)

(b)

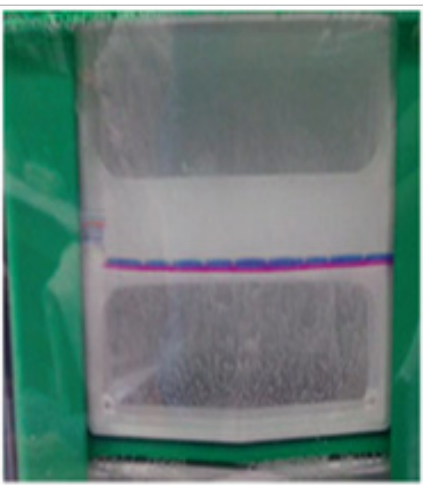

Figure 5 (a) Samples running through the stacking gel (lower voltage) (b): Samples running through the separating gel (higher voltage).

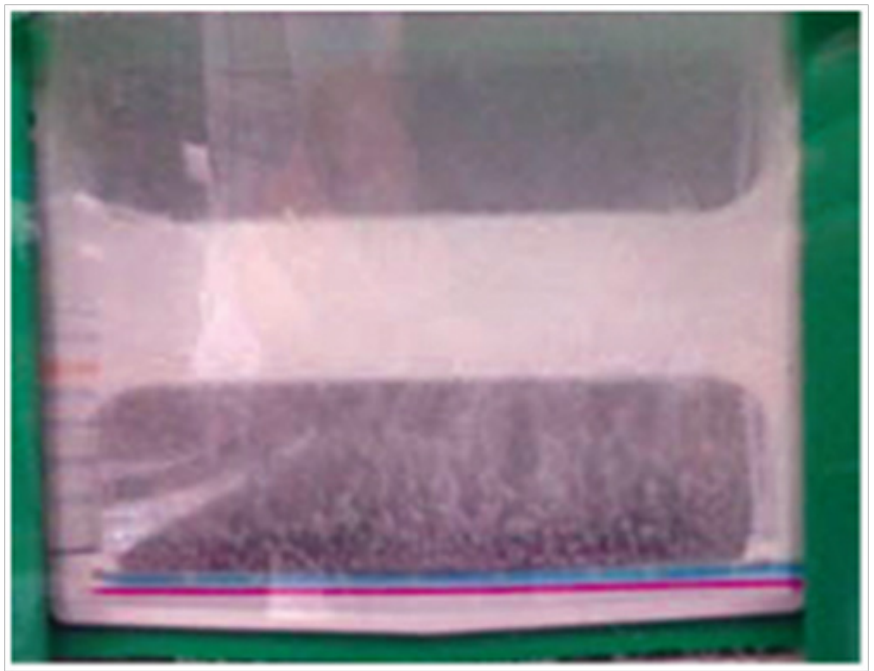

Figure 6 Running gel to the bottom of electro phoretor.

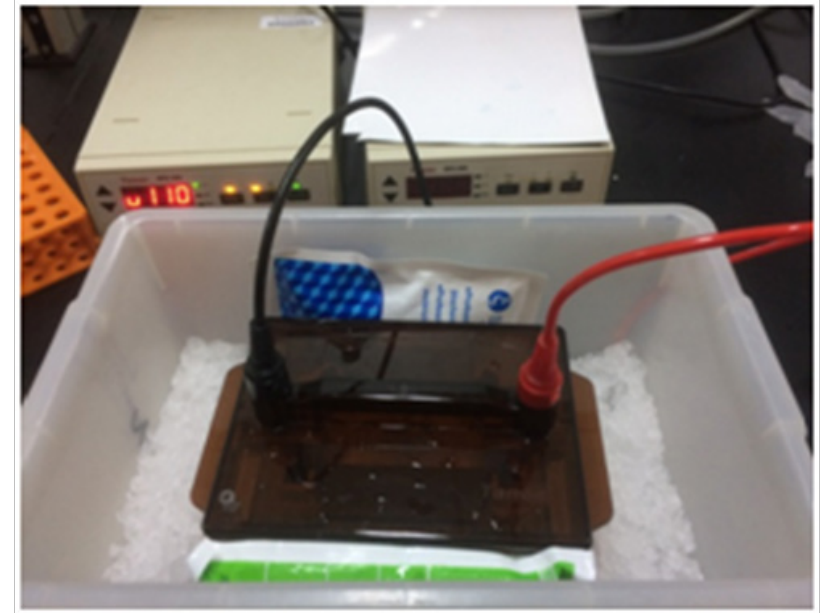

Figure 7 Transfer should be done on ice.

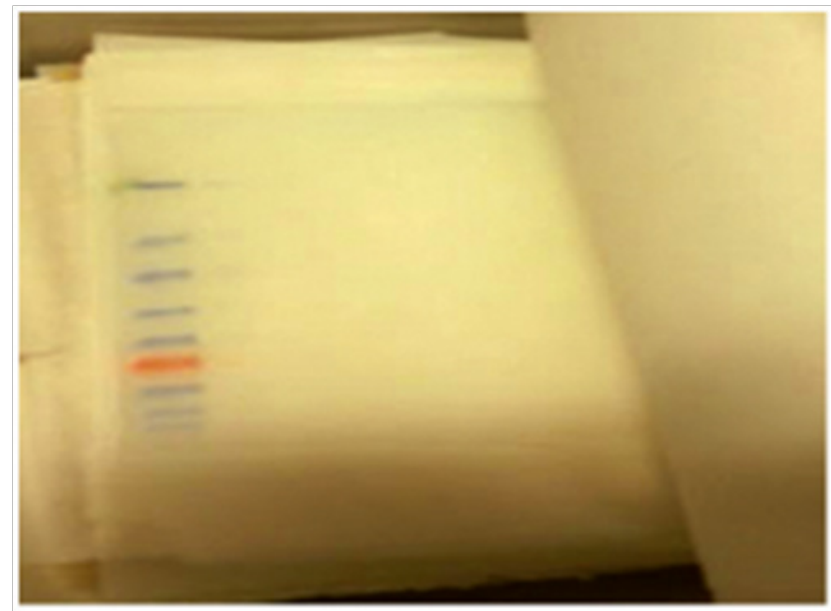

Figure 8 Membrane after transfer.

\section{Blocking and antibody incubation}

a. Block the PVDF membrane with 5\% skim milk in TBST* for 1hour.

b. Add primary antibody in $5 \%$ bovine serum albumin (BSA) and incubate it for overnight at $4^{\circ} \mathrm{C}$ on a shaker Figure 9.

c. Wash the membrane three times with TBST for 5minutes. (Tip: All washing and antibody incubation steps should be done on a shaker at room temperature to ensure even agitation).

d. After washing, add secondary antibody in 5\% skim milk in TBST, and incubate for 1 hour.

e. Wash the membrane three times with TBST for 5 minutes.

f. Prepare ECL mix or chemilliuminious assay (following the proportion of solution $\mathrm{A}$ and $\mathrm{B}$ provided by the manufacturer). Figure 10 incubates the membrane for 1-2minutes and uses a $1000 \mu \mathrm{L}$ pipette to ensure that ECL covers the top and bottom of the membrane).

g. Visualize the result in the dark room Figure 11. (Tip: If the background is too strong, reduce exposure time).

*TBST: Tris-Buffered Saline Tween-20 


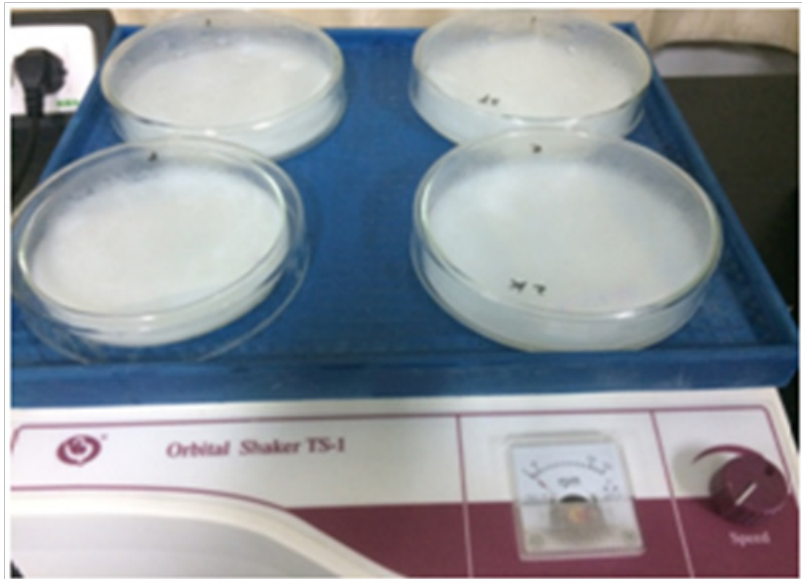

Figure 9 Use a shaker to incubate the membrane with antibody.

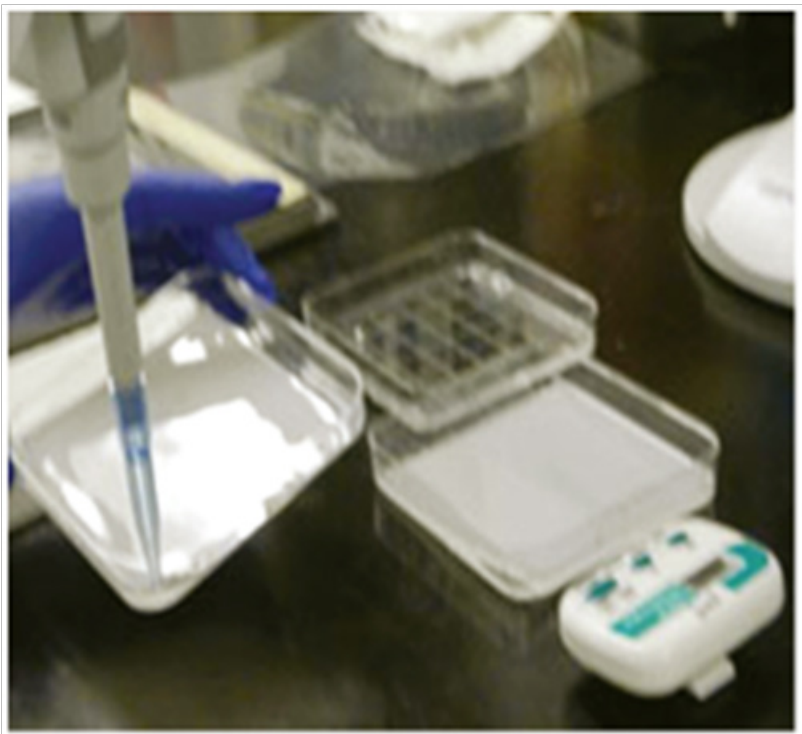

Figure 10 Incubate the membrane with ECL mix using a $1000 \mu \mathrm{L}$ pipette to help the process.

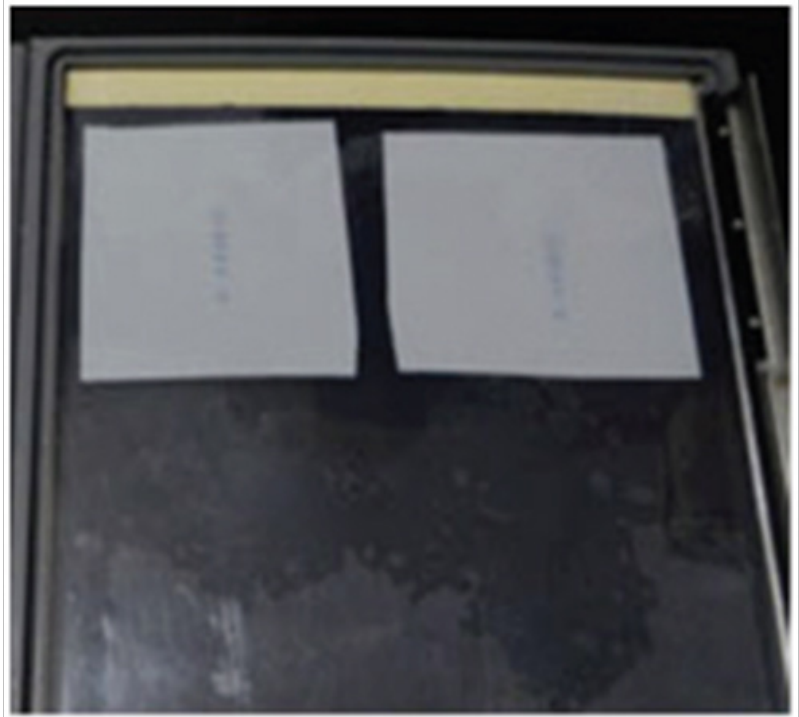

Figure I I Use the cassette to expose the membrane in the dark room.

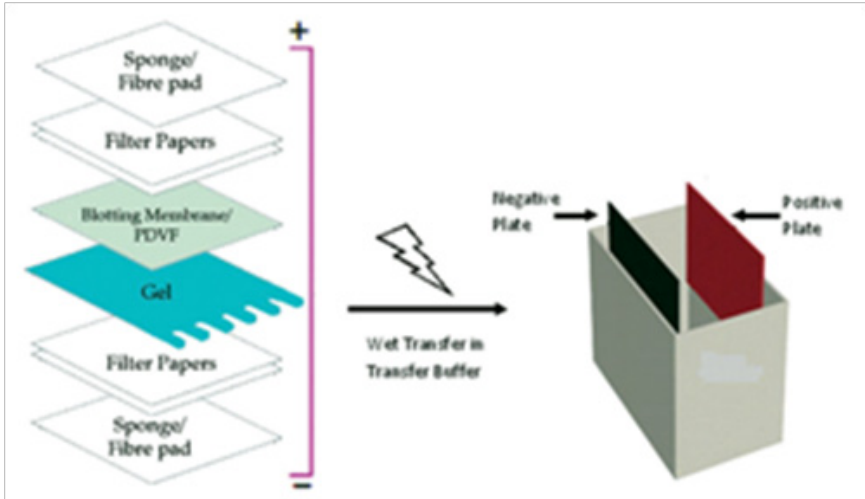

Figure 12 Assembly of a sandwich in western blot (transfer of protein bands from gel to PVDF membrane).

\section{Recipe}

a. Dissolve the following in $800 \mathrm{ml}$ of distilled $\mathrm{H}_{2} \mathrm{O}$
i. $8.8 \mathrm{~g}$ of $\mathrm{NaCl}$
ii. $0.2 \mathrm{~g}$ of $\mathrm{KCl}$

iii. $3 \mathrm{~g}$ of Tris base

b. Add 500ul of Tween-20

c. Adjust the $\mathrm{pH}$ to 7.4

d. Add distilled $\mathrm{H} 2 \mathrm{O}$ to $1 \mathrm{~L}$

e. Sterilize by filtration or autoclaving

\section{Theory}

Thus, putting it in BSA solution allows the antibody to be reused it if the blot does not give good result. The antibody can be diluted in a wash buffer, such as PBS or TBST. Washing is also very important step as it minimized background and removes unbound antibody.

\section{Sample preparation}

Western blot depends on cell lysates which is the most common form of sample used in this technique. Protein extraction should be done in a cold temperature with protease inhibitors to collect all the proteins in the cell cytosol and prevent denaturing of the proteins. Since tissue sample have a higher structural organization, therefore mechanical invention such as sonication is needed to extract the proteins. After extracting the protein, the next important step is to have a good idea of the extracted proteins concentration. This allows the researcher to ensure that the samples are being compared on an equivalent basis.

Generally spectrophotometer is used for measuring the protein concentration. This concentration value is used to measure the mass of the protein that is being loaded into each well by the relationship between concentration, mass, and volume.

After determining the appropriate volume of the sample, it is diluted with loading buffer having tracking dye so that the samples sink easily into the wells of the gel. A tracking dye (bromophenol blue) allows the researcher to see how far the separation has progressed. In order to denature the higher order structural organization of protiens, while retaining sulfide bridges, the sample is heated after being diluted into a loading buffer. Denaturing ensures that the negative charge of amino 
acids is not neutralized, enabling the protein to move in an electric field (applied during electro transfer). It is important to use positive and negative controls for the sample. For a positive control a known source of target protein, such as purified protein or a control lysate is used to confirm the identity of the protein, and the activity of the antibody. A negative control is a null cell line is used to confirm that the staining is not nonspecific in nature.

\section{Gel electrophoresis}

Two different types of agarose gel named as: stacking and separating gel are used in western blot. The stacking gel is slightly acidic having $\mathrm{pH} 6.8$ and has a lower acryl amide concentration making a porous gel, which separates protein poorly but allows them to form thin, sharply defined bands. The separating, or resolving gel, is basic with $\mathrm{pH} 8.8$, and has higher poly acryl amide content, making the gel's pores narrower. The smaller proteins are separated in this gel rapidly than larger proteins. The proteins loaded on the gel have a negative charge and will travel toward the positive electrode when a voltage is applied. Gels are usually made by pouring them between two glass and plastic plates, using the solution described in the protocol section. The wells are loaded with the samples and markers. Then, the gel is connected to the power supply and allowed to run. It is very important to regulate the voltage, as a high voltage can overheat and distort the bands on the gel, so care should be taken.

\section{Blotting}

After separating the protein mixture on the gel, the next important step is to transfer it to a membrane. This transfer is done using an electric field oriented perpendicular to the surface of the gel, causing proteins to move from gel to PVDF membrane. The membrane is placed between the gel surface and the positive electrode in a sandwich manner. The sandwich includes a sponge membrane at each end, and filter papers to protect the gel and blotting membrane Figure 12. During this process, two things are very important:

a. The placement of the membrane between the gel and the positive electrode and

b. The close contact of gel and membrane to ensure a clear image on the membrane.

The membrane must be placed as such that helps the negatively charged proteins can migrate from the gel to the membrane. This type of transfer is called electro phoretic transfer, and can be carried out in semi-dry or wet conditions. Wet conditions are usually more favorable and reliable as it is less likely to dry out the gel, and is preferred for larger proteins.

The membrane used during transferring of proteins from gel to membrane is an essential part of this process and are of two types: nitrocellulose and PVDF. Nitrocellulose is used for its high affinity for protein but does not allow the membrane to be used for re-probing. In this regard, PVDF membranes provide better reliable support and allow the blot to be re-probed and stored. However, in case of PVDF membranes the background is higher and therefore, washing is very important step for this.

\section{Washing, blocking and antibody incubation}

Washing and blocking are very important steps of western blotting, as it prevents antibodies from binding to the membrane nonspecifically. Blocking is done with 5\% BSA or nonfat dried milk diluted with TBST to reduce the effect of background. Nonfat dried milk is often preferred as it is inexpensive and widely available. However, care must be taken to choose the appropriate blocking solution because milk proteins are not compatible with all detection labels. For example, BSA blocking solutions are preferred with biotin and AP antibody labels, since milk contains casein, which is itself a phospho protein and biotin, thus interfering with the assay results. It is a good strategy to incubate the primary antibody with BSA since it is usually needed in higher amounts than the secondary antibody.

Using the label antibody usually with an enzyme such as horseradish peroxidase (HRP), the membrane is then detected by the signal. This signal is captured on a film which is usually developed in a dark room.

\section{Quantification}

It is very important to note that the data produced with a western blot is typically considered to be semi quantitative. It provides a relative comparison of protein levels, but not an absolute measure of quantity. There are two reasons; first, there are variations in loading and transfer rates between the samples in separate lanes which are different on separate blots. These differences will need to be standardized before a more precise comparison can be made. Second, the signal generated by detection is not linear across the concentration range of samples. Thus, since the signal produced is not linear, it should not be used to model the concentration.

\section{Troubleshooting and suggestions}

Western blot seems to be very simple technique but many problems can arise, leading to unexpected results. The problem can be grouped into five categories:
a. No bands
b. Unusual or unexpected bands
c. Weak signal bands
d. High background on the blot and
e. Patchy or uneven spots on the blot

Unusual or unexpected bands can be due to protease degradation. In this case it is desirable to use a fresh sample which had been kept on ice or alter the antibody. Similarly, blurry bands are often caused by high voltage or air bubbles present during transfer. In this case, it should be ensured that the gel is run at a lower voltage, and that the transfer sandwich is prepared properly. Finally, white (negative) bands on the film are due to too much protein or antibody.

No bands can also arise due to many reasons related to antibody, antigen, or buffer used. The following factors are responsible for that;
A. Improper primary and secondary antibody
B. Low concentration of antibodies or antigen
C. Prolong washing can also decrease the signals
D. Non fat dry milk

It is also important to use a shaker for all incubation, so that there is no uneven agitation during the incubation.

\section{Conclusion}

Western blot is a protein detection technique as it allows the user to detect and quantify the protein expression as well. This technical paper covered the basic protocol, the theory behind that protocol, and some troubleshooting techniques and suggestions. It will help researcher to find protein expression accurately. 


\section{Acknowledgements}

None.

\section{Conflict of interest}

The author declares no conflict of interest. 